\title{
New Convolutions for Quadratic-Phase Fourier Integral Operators and their Applications]
}

\author{
L.P. Castro, L.T. Minh and N.M. Tuan
}

\begin{abstract}
We obtain new convolutions for quadratic-phase Fourier integral operators (which include, as subcases, e.g., the fractional Fourier transform and the linear canonical transform). The structure of these convolutions is based on properties of the mentioned integral operators and takes profit of weight-functions associated with some amplitude and Gaussian functions. Therefore, the fundamental properties of that quadraticphase Fourier integral operators are also studied (including a Riemann-Lebesgue type lemma, invertibility results, a Plancherel type theorem and a Parseval type identity). As applications, we obtain new Young type inequalities, the asymptotic behaviour of some oscillatory integrals, and the solvability of convolution integral equations.
\end{abstract}

Keywords: convolution; Young inequality; oscillatory integral; convolution integral equation; fractional Fourier transform; linear canonical transform.

AMS Subject Classification: Primary 44A35; Secondary 42A38; 42A85; 43A32; 44A20; 45E10.

\section{Introduction}

The interest in having new convolutions associated with integral operators is wide and based on both theoretical and applied aspects. Outside mathematics, the use of different types of convolutions is very diverse, ranging, e.g., from signal processing to neural networks. Within mathematics, it is also very profitable to construct new convolutions that will facilitate the identification of new factorization properties to decouple the convolutions into a (weighted) product of integrals. Typically, this decoupling has strong consequences and originates new results in different branches of mathematics (e.g., in harmonic analysis and differential equations). This last aspect will be exhibited in here, for a large class of integral operators, with

\footnotetext{
${ }^{1}$ Accepted author's manuscript (AAM) of [L.P. Castro, L.T. Minh, N.M. Tuan: Mediterr. J. Math. (2018) 15:13. DOI: 10.1007/s00009-017-1063-y].

The final publication is available at Springer via https://doi.org/10.1007/s00009-017-1063-y and http://rdcu.be/DWRU
} 
consequences that will be exemplified for three different topics: (i) Young type inequalities, (ii) asymptotic behaviour of oscillatory integrals, and (iii) the solvability of classes of integral equations.

A diversity of convolutions which are suitable for other integral operators can be found in several recent publications. For other convolutions and integral operators, while not being exhaustive, we refer the reader to [1, 2, 3, 8, 12, 13, 14, 15, 16, 17, 18, 22, 25, 26, 28,. In addition, it is relevant to have in mind that the factorization property of convolutions is crucial in solving corresponding convolution type equations [6, 7, 11, 25]. It is also clear that convolution type equations are very often used in the modelling of a broad range of different problems (cf. [9, 10]), and so additional knowledge on their solvability is very welcome.

Throughout this paper, for parameters $a, b, c, d, e \in \mathbb{R}$ (with $b \neq 0$ ), we take

$$
Q_{(a, b, c, d, e)}(x, y):=a x^{2}+b x y+c y^{2}+d x+e y
$$

to be the quadratic-phase function within the kernel of our integral operator. In what follows, for shortening formulas, we will also use the notation $Q_{(a-e)}(x, y):=Q_{(a, b, c, d, e)}(x, y)$. Besides this, we shall also write $Q_{(a-c)}(x, y):=Q_{(a, b, c, 0,0)}(x, y)$. So, this allows us to introduce the integral operator $\mathbb{Q}$ defined by

$$
(\mathbb{Q} f)(x):=\frac{1}{\sqrt{2 \pi}} \int_{\mathbb{R}} e^{i Q_{(a-e)}(x, y)} f(y) d y,
$$

where $f \in L^{1}(\mathbb{R})$ or $f \in L^{2}(\mathbb{R})$, and that we will denominate by quadratic-phase Fourier integral operator.

Let us make a brief discussion on the integral operator $\mathbb{Q}$ by comparing it with other well-known operators. In first place, we would like to notice that when $a=c=d=e=0$ and $b= \pm 1, \mathbb{Q}$ is simply the well-known Fourier and inverse Fourier integral transforms, respectively. Secondly, when $d=e=0$, the kernel generated by (1.1) includes the kernel of the linear canonical transform as well as of the one of the fractional Fourier transform. Typically, it is clear that the constant factors incorporated in the integral operators are considered in view of the final purposes and problems where the operators are used. Still within in the last comparison, and as about the constant factor appearing in (1.2), there is a difference between our concept of quadratic-phase Fourier integral operator and the most frequent choices of constant factors for the linear canonical transform and fractional Fourier transform. In our case, the factor $1 / \sqrt{2 \pi}$ is chosen intentionally since it ensures consequent convenient computations involving the quadratic-phase Fourier integral operator, as we shall see later on. The constant $\sqrt{-i}$ typically chosen in the particular case of linear canonical 
transform, and $\sqrt{(1-i \cot (\alpha)) / 2 \pi}$ for the fractional Fourier transform, are more convenient in view of the particular properties of those cases.

The paper is divided into four sections and organized as follows. Section 2 presents some basic theorems for the integral operator $\mathbb{Q}$ such as the Riemann-Lebesgue lemma, inversion formula, Plancherel's extension, Parseval identity. Section 3 provides new convolution theorems which, in particular cases, turn out to be convolution theorems for the linear canonical transform, fractional Fourier transform and Fourier transform. In Section 4 we apply the obtained results in order to derive Young's convolution inequalities for the proposed convolutions, the asymptotic behaviour of a class of oscillatory integrals, and the solvability of classes of convolution integral equations.

\section{Basic Properties of the Integral Operator}

In this section, as a preliminary step to the main content, we will study some basic properties of the integral operator $\mathbb{Q}$. This will include its mapping properties, as well as a corresponding inversion formula, a Plancherel's extension and a Parseval identity.

Let us denote by $\mathcal{S}$ the Schwartz space, and by $C_{0}(\mathbb{R})$ the Banach space of all continuous functions on $\mathbb{R}$ that vanish at infinity, endowed with the supremum norm $\|\cdot\|_{\infty}$. Moreover, in $L^{1}(\mathbb{R})$ we will be using the norm $\|\cdot\|_{1}$ defined by

$$
\|f\|_{1}:=\frac{1}{\sqrt{2 \pi}} \int_{\mathbb{R}}|f(y)| d y
$$

where the factor $1 / \sqrt{2 \pi}$ is here considered just to obtain more direct computations later on. In the case of $1<p<\infty$, the space $L^{p}(\mathbb{R})$ will be endowed with the norm

$$
\|f\|_{p}:=\left(\int_{\mathbb{R}}|f(y)|^{p} d y\right)^{\frac{1}{p}} .
$$

Lemma 2.1 (Riemann-Lebesgue lemma). If $f \in L^{1}(\mathbb{R})$ then $\mathbb{Q} f \in C_{0}(\mathbb{R})$, and $\|\mathbb{Q} f\|_{\infty} \leq$ $\|f\|_{1}$.

Proof. Since $\left|e^{i Q_{(a-e)}(x, y)}\right|=1$, it is clear that

$$
\begin{aligned}
\|\mathbb{Q} f\|_{\infty} & =\sup _{x \in \mathbb{R}}|(\mathbb{Q} f)(x)|=\sup _{x \in \mathbb{R}} \frac{1}{\sqrt{2 \pi}}\left|\int_{\mathbb{R}} e^{i Q_{(a-e)}(x, y)} f(y) d y\right| \\
& \leq \sup _{x \in \mathbb{R}} \frac{1}{\sqrt{2 \pi}} \int_{\mathbb{R}}\left|e^{i Q_{(a-e)}(x, y)}\right||f(y)| d y=\|f\|_{1} .
\end{aligned}
$$


In addition, choosing $g(y):=e^{i\left(c y^{2}+e y\right)} f(y)$ it is clear that $g \in L^{1}(\mathbb{R})$ if and only if $f \in L^{1}(\mathbb{R})$. Therefore, using the classic Riemann-Lebesgue lemma, we derive that

$$
|(\mathbb{Q} f)(x)|=\frac{\left|e^{i\left(a x^{2}+d x\right)}\right|}{\sqrt{2 \pi}}\left|\int_{\mathbb{R}} e^{i b x y} g(y) d y\right|=\frac{1}{\sqrt{2 \pi}}\left|\int_{\mathbb{R}} e^{i b x y} g(y) d y\right| \rightarrow 0,
$$

as $x \rightarrow \infty$, and the proof of the lemma is complete.

The next lemma is known as a version of the Nyquist-Shannon sampling theorem, and will be useful for proving Theorem 2.3 .

Lemma 2.2 (cf., e.g., Theorem 12, [24]). The formula

$$
\frac{1}{2}[f(x+0)+f(x-0)]=\lim _{\lambda \rightarrow \infty} \frac{1}{\pi} \int_{-\infty}^{+\infty} f(t) \frac{\sin \lambda(x-t)}{x-t} d t
$$

holds true if $\frac{f(x)}{1+|x|}$ belongs to $L^{1}(\mathbb{R})$.

Theorem 2.3 (Inversion theorem). If $f \in L^{1}(\mathbb{R})$ and $\mathbb{Q} f \in L^{1}(\mathbb{R})$, then

$$
f(x)=\frac{b}{\sqrt{2 \pi}} \int_{\mathbb{R}}(\mathbb{Q} f)(y) e^{-i Q_{(a-e)}(y, x)} d y,
$$

for almost every $x \in \mathbb{R}$.

Proof. First, let us prove the inversion formula for $f \in \mathcal{S}$. In this case, by Lemma 2.2 and direct computations, we have

$$
\begin{aligned}
\frac{b}{\sqrt{2 \pi}} \int_{\mathbb{R}}(\mathbb{Q} f)(y) e^{-i Q_{(a-e)}(y, x)} d y \\
\quad=\frac{b}{2 \pi} \lim _{\lambda \rightarrow+\infty} \int_{-\lambda}^{\lambda} \int_{\mathbb{R}} e^{-i Q_{(a-e)}(y, x)} e^{i Q_{(a-e)}(y, u)} f(u) d u d y \\
\quad=\frac{b}{2 \pi} e^{-i\left(c x^{2}+e x\right)} \int_{\mathbb{R}} f(u) e^{i\left(c u^{2}+e u\right)} d u \lim _{\lambda \rightarrow+\infty} \int_{-\lambda}^{\lambda} e^{-i b y(x-u)} d y \\
\quad=\frac{1}{\pi} e^{-i\left(c x^{2}+e x\right)} \lim _{\lambda \rightarrow+\infty} \int_{\mathbb{R}} f(u) e^{i\left(c u^{2}+e u\right)} \frac{\sin b \lambda(x-u)}{x-u} d u \\
=e^{-i\left(c x^{2}+e x\right)} f(x) e^{i\left(c x^{2}+e x\right)}=f(x) .
\end{aligned}
$$

Thus, $\mathbb{Q}$ is a one-to-one, linear and continuous operator from $\mathcal{S}$ onto $\mathcal{S}$ (with a continuous inverse).

We now assume that $f \in L^{1}(\mathbb{R})$, and let $g \in \mathcal{S}$. A direct computation gives us

$$
\int_{\mathbb{R}} f(x)(\mathbb{Q} g)(x) d x=\int_{\mathbb{R}} g(y)(\mathbb{Q} f)(y) d y .
$$


Using this identity and (2.1), for $g \in \mathcal{S}$, we have

$$
\begin{aligned}
\int_{\mathbb{R}} f(x)(\mathbb{Q} g)(x) d x & =\frac{b}{\sqrt{2 \pi}} \int_{\mathbb{R}}\left(\int_{\mathbb{R}} e^{-i Q_{(a-e)}(y, x)}(\mathbb{Q} g)(x) d x\right)(\mathbb{Q} f)(y) d y \\
& =\int_{\mathbb{R}}(\mathbb{Q} g)(x)\left(\frac{b}{\sqrt{2 \pi}} \int_{\mathbb{R}} e^{-i Q_{(a-e)}(y, x)}(\mathbb{Q} f)(y) d y\right) d x \\
& =\int_{\mathbb{R}} f_{0}(x)(\mathbb{Q} g)(x) d x
\end{aligned}
$$

where

$$
f_{0}(x):=\frac{b}{\sqrt{2 \pi}} \int_{\mathbb{R}}(\mathbb{Q} f)(y) e^{-i Q_{(a-e)}(y, x)} d y .
$$

By (2.1) the function $\mathbb{Q} g$ covers all $\mathcal{S}$ when $g$ runs in $\mathcal{S}$. Therefore, $\int_{\mathbb{R}}\left(f_{0}(x)-f(x)\right) \Phi(x) d x$ $=0$ for every $\Phi \in \mathcal{S}$. Since $\mathcal{S}$ is dense in $L^{1}(\mathbb{R})$, we obtain that $f_{0}(x)-f(x)=0$ for almost every $x \in \mathbb{R}$, as desired.

The uniqueness property below is an immediate consequence of Theorem 2.3 .

Corollary 2.4 (Uniqueness). If $f \in L^{1}(\mathbb{R})$ and $\mathbb{Q} f=0$, then $f=0$.

In what follows we will be denoting the inverse operator of $\mathbb{Q}$ by $\mathbb{Q}^{-1}$ :

$$
\left(\mathbb{Q}^{-1} g\right)(x):=\frac{b}{\sqrt{2 \pi}} \int_{\mathbb{R}}(\mathbb{Q} f)(y) e^{-i Q_{(a-e)}(y, x)} d y
$$

Theorem 2.5 (Plancherel theorem). There is a linear isomorphic operator $\overline{\mathbb{Q}}: L^{2}(\mathbb{R}) \rightarrow$ $L^{2}(\mathbb{R})$ which is uniquely determined by the requirement that $\overline{\mathbb{Q}} f=\mathbb{Q} f$ for every $f \in \mathcal{S}$. The inverse operator is also uniquely determined by having $\overline{\mathbb{Q}}^{-1} f=\mathbb{Q}^{-1} f$ for every $f \in \mathcal{S}$. Proof. Recall that $\mathcal{S}$ is dense in $L^{2}(\mathbb{R})$. Thus, as the map $f \mapsto \mathbb{Q} f$ is continuous (relative to the $L^{2}$-norm) from the dense subspace $\mathcal{S}$ of $L^{2}(\mathbb{R})$ onto $\mathcal{S}$, it has a unique continuous extension $\overline{\mathbb{Q}}: L^{2}(\mathbb{R}) \rightarrow L^{2}(\mathbb{R})$. Theorem 2.5 is proved.

Thanks to the uniqueness of the extension operator, one can formulate another theorem in a more detailed way by exhibiting the explicit form of the operator in $L^{2}$.

Theorem 2.6 (Plancherel theorem). Let $f$ be a complex-valued function in $L^{2}(\mathbb{R})$ and let

$$
\mathbb{Q}(x, k):=\frac{1}{\sqrt{2 \pi}} \int_{|y|<k} f(y) e^{i Q_{(a-e)}(x, y)} d y .
$$

Then, as $k \rightarrow \infty, \mathbb{Q}(x, k)$ converges strongly (over $\mathbb{R})$ to a function, say $\mathbb{Q} f$, of $L^{2}(\mathbb{R})$; reciprocally,

$$
f(x, k):=\frac{b}{\sqrt{2 \pi}} \int_{|y|<k} \mathbb{Q} f(y) e^{-i Q_{(a-e)}(y, x)} d y .
$$

converges strongly to $f$. 
Theorem 2.7 (Parseval type identity). (i) For any $f, g \in L^{2}(\mathbb{R})$, the following identity holds

$$
\langle\mathbb{Q} f, \mathbb{Q} g\rangle=\frac{1}{|b|}\langle f, g\rangle,
$$

where $\langle\cdot, \cdot\rangle$ denotes the usual inner product in $L^{2}(\mathbb{R})$ given by $\langle f, g\rangle:=\int_{\mathbb{R}} f(x) \overline{g(x)} d x$. In the special case of $f=g$, we then have

$$
\|\mathbb{Q} f\|_{2}^{2}=\frac{1}{|b|}\|f\|_{2}^{2}
$$

(ii) If $|b|=1$, then $\mathbb{Q}$ defines a unitary operator in $L^{2}(\mathbb{R})$.

Proof. Consider $b>0$. One can prove this theorem by different ways, directly or indirectly. Directly, by Lemma 2.2 and simple computations, we have

$$
\begin{aligned}
\langle\mathbb{Q} f, \mathbb{Q} g\rangle & =\int_{\mathbb{R}}(\mathbb{Q} f)(x) \overline{(\mathbb{Q} g)(x)} d x \\
& =\frac{1}{2 \pi} \int_{\mathbb{R}} \int_{\mathbb{R}} \int_{\mathbb{R}} e^{i Q_{(a-e)}(x, y)} e^{-i Q_{(a-e)}(x, u)} f(y) \overline{g(u)} d y d u d x \\
& =\frac{1}{2 \pi} \int_{\mathbb{R}} \int_{\mathbb{R}} e^{i\left(c y^{2}+e y\right)} e^{-i\left(c u^{2}+e u\right)} f(y) \overline{g(u)} d y d u \int_{\mathbb{R}} e^{i b x(y-u)} d x \\
& =\frac{1}{2 \pi} \int_{\mathbb{R}} \int_{\mathbb{R}} e^{i\left(c y^{2}+e y\right)} e^{-i\left(c u^{2}+e u\right)} f(y) \overline{g(u)} d y d u\left(\lim _{\lambda \rightarrow \infty} \int_{-\lambda}^{\lambda} e^{i b x(y-u)} d x\right) \\
& =\frac{1}{b} \int_{\mathbb{R}} e^{i\left(c y^{2}+e y\right)} f(y)\left(\lim _{\lambda \rightarrow \infty} \frac{1}{\pi} \int_{\mathbb{R}}\left[e^{-i\left(c u^{2}+e u\right)} \overline{g(u)} \frac{\sin b \lambda(y-u)}{y-u} d u\right) d y\right. \\
& =\frac{1}{b} \int_{\mathbb{R}} e^{i\left(c y^{2}+e y\right)} f(y) e^{-i\left(c y^{2}+e y\right)} \overline{g(y)} d y=\frac{1}{b} \int_{\mathbb{R}} f(y) \overline{g(y)} d y=\frac{1}{b}\langle f, g\rangle .
\end{aligned}
$$

Similarly, if $b<0$ then $\langle\mathbb{Q} f, \mathbb{Q} g\rangle=\frac{1}{-b}\langle f, g\rangle$. Thus, proposition (i) is proved. Having now in mind that $\mathbb{Q}$ is surjective (cf. Theorem 2.5), and proposition (i), it follows that (ii) holds true.

\section{New Convolutions}

We will introduce new convolutions somehow associated with the integral operator $\mathbb{Q}$ which will exhibit very significant factorization identities. Later on, in the next section, we will exemplify the use of such convolutions and their factorizations.

In what follows, we will use the following well-known identity

$$
\frac{1}{\sqrt{2 \pi}} \int_{\mathbb{R}} e^{i x t} e^{-k t^{2}} d t=\frac{1}{\sqrt{2 k}} e^{-\frac{1}{4 k} x^{2}} \quad(k>0)
$$

for every $x \in \mathbb{R}$ (see [21, 24]). 
Theorem 3.1. If $f, g \in L^{1}(\mathbb{R})$ and $\Omega_{1}(x):=e^{-\frac{1}{2} x^{2}-a i x^{2}}$, then the new element $f \stackrel{\Omega_{1}}{\star} g$ below introduced defines a convolution followed by the norm inequality and its factorization identity:

$$
\begin{aligned}
& \left(f \underset{\mathbb{Q}}{\stackrel{\Omega_{1}}{\star}} g\right)(x):=\frac{b}{2 \pi} \int_{\mathbb{R}} \int_{\mathbb{R}} f(u) g(v) e^{i\left(c u^{2}+c v^{2}-c x^{2}+e u+e v-e x\right)-\frac{(b x-b u-b v-d)^{2}}{2}} d u d v, \\
& \left\|f \stackrel{\Omega_{1}}{\stackrel{\Omega_{1}}{\star}} g\right\|_{1} \leq\|f\|_{1}\|g\|_{1}, \quad \mathbb{Q}\left(f \underset{\mathbb{Q}}{\stackrel{\Omega_{1}}{\star}} g\right)(x)=\Omega_{1}(x)(\mathbb{Q} f)(x)(\mathbb{Q} g)(x) .
\end{aligned}
$$

Proof. First, we prove the norm inequality. Performing the change of variables $t:=b x-$ $b u-b v-d$ and using (3.1), we obtain

$$
\begin{aligned}
\left\|f \underset{\mathbb{Q}}{\stackrel{\Omega_{1}}{\star}} g\right\|_{1} & \leq \frac{|b|}{(2 \pi)^{3 / 2}} \int_{\mathbb{R}}|f(u)| d u \int_{\mathbb{R}}|g(v)| d v \int_{\mathbb{R}} e^{-\frac{(b x-b u-b v-d)^{2}}{2}} d x \\
& =\frac{\|f\|_{1}\|g\|_{1}}{\sqrt{2 \pi}} \int_{\mathbb{R}} e^{-\frac{t^{2}}{2}} d t=\|f\|_{1}\|g\|_{1},
\end{aligned}
$$

which proves the norm inequality. We shall now prove the factorization identity. Using (3.1), we have

$$
\begin{aligned}
& \Omega_{1}(x)(\mathbb{Q} f)(x)(\mathbb{Q} g)(x) \\
& =e^{-\frac{1}{2} x^{2}-a i x^{2}} \frac{1}{\sqrt{2 \pi}} \int_{\mathbb{R}} e^{i Q_{(a-e)}(x, u)} f(u) d u \frac{1}{\sqrt{2 \pi}} \int_{\mathbb{R}} e^{i Q_{(a-e)}(x, v)} g(v) d v \\
& =e^{-a i x^{2}} \frac{1}{\sqrt{2 \pi}} \int_{\mathbb{R}} e^{-\frac{1}{2} t^{2}+i x t} d t \frac{1}{2 \pi} \int_{\mathbb{R}} \int_{\mathbb{R}} e^{i Q_{(a-e)}(x, u)} e^{i Q_{(a-e)}(x, v)} f(u) g(v) d u d v \\
& =\frac{1}{2 \pi \sqrt{2 \pi}} \int_{\mathbb{R}} \int_{\mathbb{R}} \int_{\mathbb{R}} e^{i\left(a x^{2}+c u^{2}+c v^{2}+b x\left(u+v+\frac{t}{b}+\frac{d}{b}\right)+d x+e u+e v\right)} e^{-\frac{1}{2} t^{2}} f(u) g(v) d u d v d t .
\end{aligned}
$$

Let $u=u, v=v$ and $s=u+v+\frac{t}{b}+\frac{d}{b}$. We then have

$$
\begin{aligned}
& \Omega_{1}(x)(\mathbb{Q} f)(x)(\mathbb{Q} g)(x)=\frac{b}{2 \pi \sqrt{2 \pi}} \int_{\mathbb{R}} \int_{\mathbb{R}} \int_{\mathbb{R}} e^{i Q_{(a-e)}(x, s)} \times \\
& e^{i\left(c u^{2}+c v^{2}-c s^{2}+e u+e v-e s\right)-\frac{(b s-b u-b v-d)^{2}}{2}} f(u) g(v) d u d v d s \\
& =\frac{1}{\sqrt{2 \pi}} \int_{\mathbb{R}} e^{i Q_{(a-e)}(x, s)}\left\{\frac{b}{2 \pi} \int_{\mathbb{R}} \int_{\mathbb{R}} f(u) g(v) \times\right. \\
& \left.e^{i\left(c u^{2}+c v^{2}-c s^{2}+e u+e v-e s\right)-\frac{(b s-b u-b v-d)^{2}}{2}} d u d v\right\} d s \\
& =\frac{1}{\sqrt{2 \pi}} \int_{\mathbb{R}} e^{i Q_{(a-e)}(x, s)}\left(f \stackrel{\Omega_{1}}{\stackrel{\mathbb{Q}}{\star}} g\right)(s) d s=\mathbb{Q}\left(f \underset{\mathbb{Q}}{\stackrel{\Omega_{1}}{\star}} g\right)(x) .
\end{aligned}
$$

The theorem is proved.

Remark 3.2. (a) When $a=c=d=e=0$, and $b= \pm 1, \mathbb{Q}$ is the well-known Fourier transform and inverse Fourier transform, respectively, and $\Omega_{1}(x)=e^{-\frac{1}{2} x^{2}}$. Then, the convolution 
in (3.2) takes the form

$$
\left(f \stackrel{\Omega_{1}}{\stackrel{\Omega_{\mathbb{Q}}}{\star}} g\right)(x)=\frac{ \pm 1}{\sqrt{2 \pi}} \int_{\mathbb{R}} \int_{\mathbb{R}} e^{-\frac{(x-u-v)^{2}}{2}} f(u) g(v) d u d v,
$$

and so are convolutions associated with the Fourier transform.

(b) Let $a=c=\cot (\alpha) / 2, b=-\sec (\alpha)$. In such a particular case we see that $\mathbb{Q}$ is simply the fractional Fourier transform, and (3.2) takes the form

$$
\left(f \stackrel{\Omega_{1}}{\stackrel{\Omega_{\mathbb{Q}}}{\star}} g\right)(x)=\frac{b}{2 \pi} \int_{\mathbb{R}} \int_{\mathbb{R}} e^{i a\left(u^{2}+v^{2}-x^{2}\right)-\frac{b^{2}}{2}(x-u-v)^{2}} f(u) g(v) d u d v,
$$

which is therefore a convolution associated with the fractional Fourier transform.

(c) Let $d=e=0$. Then, $\mathbb{Q}$ is the linear canonical transform and (3.2) turns to be

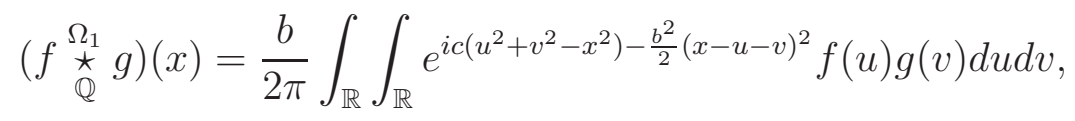

being therefore a convolution associated with the linear canonical transform.

Theorem 3.3. If $f, g \in L^{1}(\mathbb{R})$ and $\Omega_{2}(x):=e^{-\frac{1}{2} x^{2}-a i x^{2}-\text { dix }}$, then the following element $f \underset{\mathbb{Q}}{\otimes} g$ is a convolution followed by its norm inequality and factorization identity:

$$
\begin{gathered}
\left(f \underset{\mathbb{Q}}{\stackrel{\Omega_{2}}{\otimes}} g\right)(x):=\frac{b}{2 \pi} \int_{\mathbb{R}} \int_{\mathbb{R}} e^{i\left(c u^{2}+c v^{2}-c x^{2}+e u+e v-e x\right)-\frac{(b x-b u-b v)^{2}}{2}} f(u) g(v) d u d v, \\
\left\|f \underset{\mathbb{Q}}{\Omega_{2}} g\right\|_{1} \leq\|f\|_{1}\|g\|_{1}, \quad \mathbb{Q}(f \underset{\mathbb{Q}}{\otimes} g)(x)=\Omega_{2}(x)(\mathbb{Q} f)(x)(\mathbb{Q} g)(x) .
\end{gathered}
$$

Proof. The proof of the norm inequality can be obtained in the same way as in the case of convolution (3.2), and so it is here omitted. For proving the factorization identity, we start by interpreting the right-hand side of the factorization identity:

$$
\begin{aligned}
& \Omega_{2}(x)(\mathbb{Q} f)(x)(\mathbb{Q} g)(x) \\
& =e^{-\frac{1}{2} x^{2}-a i x^{2}-d i x} \frac{1}{\sqrt{2 \pi}} \int_{\mathbb{R}} e^{i Q_{(a-e)}(x, u)} f(u) d u \frac{1}{\sqrt{2 \pi}} \int_{\mathbb{R}} e^{i Q_{(a-e)}(x, v)} g(v) d v \\
& =e^{-a i x^{2}-d i x} \frac{1}{\sqrt{2 \pi}} \int_{\mathbb{R}} e^{-\frac{1}{2} t^{2}+i x t} d t \frac{1}{\sqrt{2 \pi}} \int_{\mathbb{R}} e^{i Q_{(a-e)}(x, u)} f(u) d u \frac{1}{\sqrt{2 \pi}} \int_{\mathbb{R}} e^{i Q_{(a-e)}(x, v)} g(v) d v \\
& =\frac{1}{2 \pi \sqrt{2 \pi}} \int_{\mathbb{R}} \int_{\mathbb{R}} \int_{\mathbb{R}} e^{i\left(a x^{2}+c u^{2}+c v^{2}+b x\left(u+v+\frac{t}{b}\right)+d x+e u+e v\right)} e^{-\frac{1}{2} t^{2}} f(u) g(v) d u d v d t .
\end{aligned}
$$

Then, taking $u=u, v=v$ and $s=u+v+\frac{t}{b}$, we obtain

$$
\begin{array}{r}
\Omega_{2}(x)(\mathbb{Q} f)(x)(\mathbb{Q} g)(x)=\frac{b}{2 \pi \sqrt{2 \pi}} \int_{\mathbb{R}} \int_{\mathbb{R}} \int_{\mathbb{R}} e^{i Q_{(a-e)}(x, s)} \times \\
e^{i\left(c u^{2}+c v^{2}-c s^{2}+e u+e v-e s\right)-\frac{(b s-b u-b v)^{2}}{2}} f(u) g(v) d u d v d s
\end{array}
$$




$$
\begin{aligned}
= & \frac{1}{\sqrt{2 \pi}} \int_{\mathbb{R}} e^{i Q_{(a-e)}(x, s)}\left\{\frac{b}{2 \pi} \int_{\mathbb{R}} \int_{\mathbb{R}} f(u) g(v) \times\right. \\
& \left.e^{i\left(c u^{2}+c v^{2}-c s^{2}+e u+e v-e s\right)-\frac{(b s-b u-b v)^{2}}{2}} d u d v\right\} d s \\
= & \frac{1}{\sqrt{2 \pi}} \int_{\mathbb{R}} e^{i Q_{(a-e)}(x, s)}\left(f{\underset{\mathbb{Q}}{\Omega_{2}}}^{\otimes} g\right)(s) d s=\mathbb{Q}\left(f{\stackrel{\mathbb{Q}}{\Omega_{2}}}_{\mathbb{Q}} g\right)(x) .
\end{aligned}
$$

The theorem is proved.

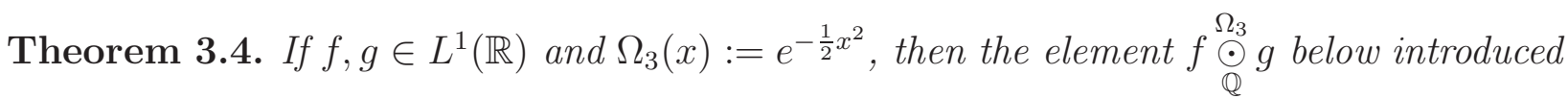
defines a convolution followed by a norm inequality and its factorization identity:

$$
\begin{aligned}
& \left(f \underset{\mathbb{Q}}{\stackrel{\Omega_{3}}{\odot}} g\right)\left(\frac{x}{\sqrt{2}}\right):=\frac{b}{\sqrt{2} \pi} \int_{\mathbb{R}} \int_{\mathbb{R}} e^{i\left(c u^{2}+c v^{2}-c \frac{x^{2}}{2}+e u+e v-\frac{e x}{\sqrt{2}}\right)-\frac{(b x-b u-b v-2 d+d \sqrt{2})^{2}}{2}} f(u) g(v) d u d v,
\end{aligned}
$$

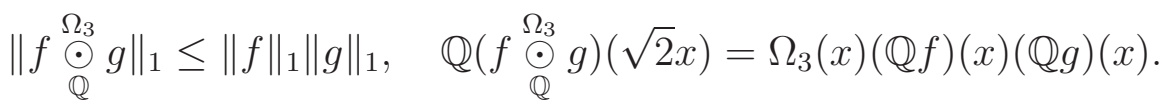

Proof. The norm inequality can be deduced in the same way as above, and so we omit its proof. Now, we realize that

$$
\begin{aligned}
& \Omega_{3}(x)(\mathbb{Q} f)(x)(\mathbb{Q} g)(x) \\
& =e^{-\frac{1}{2} x^{2}} \frac{1}{\sqrt{2 \pi}} \int_{\mathbb{R}} e^{i Q_{(a-e)}(x, u)} f(u) d u \frac{1}{\sqrt{2 \pi}} \int_{\mathbb{R}} e^{i Q_{(a-e)}(x, v)} g(v) d v \\
& =\frac{1}{\sqrt{2 \pi}} \int_{\mathbb{R}} e^{-\frac{1}{2} t^{2}+i x t} d t \frac{1}{2 \pi} \int_{\mathbb{R}} \int_{\mathbb{R}} e^{i Q_{(a-e)}(x, u)} e^{i Q_{(a-e)}(x, v)} f(u) g(v) d u d v \\
& =\frac{1}{2 \pi \sqrt{2 \pi}} \int_{\mathbb{R}} \int_{\mathbb{R}} \int_{\mathbb{R}} e^{i\left(2 a x^{2}+c u^{2}+c v^{2}+b x\left(u+v+\frac{t}{b}+\frac{2 d}{b}-\frac{d \sqrt{2}}{b}\right)+d \sqrt{2} x+e u+e v\right)} e^{-\frac{1}{2} t^{2}} f(u) g(v) d u d v d t .
\end{aligned}
$$

Then, taking $u=u, v=v$ and $s=u+v+\frac{t}{b}+\frac{2 d}{b}-\frac{d \sqrt{2}}{b}$, we obtain

$$
\begin{aligned}
& \Omega_{3}(x)(\mathbb{Q} f)(x)(\mathbb{Q} g)(x)=\frac{b}{2 \pi \sqrt{2 \pi}} \int_{\mathbb{R}} \int_{\mathbb{R}} \int_{\mathbb{R}} e^{i Q_{(a-e)}\left(\sqrt{2} x, \frac{s}{\sqrt{2}}\right)} f(u) g(v) \times \\
& e^{i\left(c u^{2}+c v^{2}-c \frac{s^{2}}{2}+e u+e v-\frac{e s}{\sqrt{2}}\right)-\frac{(b s-b u-b v-2 d+d \sqrt{2})^{2}}{2}} d u d v d s \\
& =\frac{1}{\sqrt{2 \pi}} \int_{\mathbb{R}} e^{i Q_{(a-e)}\left(\sqrt{2} x, \frac{s}{\sqrt{2}}\right)}\left\{\frac{b}{\sqrt{2} \pi} \int_{\mathbb{R}} \int_{\mathbb{R}} f(u) g(v) \times\right. \\
& \left.e^{i\left(c u^{2}+c v^{2}-c \frac{s^{2}}{2}+e u+e v-\frac{e s}{\sqrt{2}}\right)-\frac{(b s-b u-b v-2 d+d \sqrt{2})^{2}}{2}} d u d v\right\} d\left(\frac{s}{\sqrt{2}}\right) \\
& =\frac{1}{\sqrt{2 \pi}} \int_{\mathbb{R}} e^{i Q_{(a-e)}\left(\sqrt{2} x, \frac{s}{\sqrt{2}}\right)}(f \underset{\mathbb{Q}}{\odot} g)\left(\frac{s}{\sqrt{2}}\right) d\left(\frac{s}{\sqrt{2}}\right)=\mathbb{Q}(f \underset{\mathbb{Q}}{\odot} g)(\sqrt{2} x) .
\end{aligned}
$$

The theorem is proved. 
For shortness of notation, let us consider $\mathcal{E}_{\mathrm{ch}}(t):=e^{-i\left(a t^{2}+d t\right)}$ and $\mathcal{E}_{\mathrm{gd}}(t):=e^{-\frac{|b|}{2} t^{2}}$, where $\mathcal{E}_{\mathrm{ch}}, \mathcal{E}_{\mathrm{gd}}$ are the chirp and Gaussian functions, respectively. Moreover, let us also define $\Omega_{4}(t):=\mathcal{E}_{\mathrm{ch}}(t) \cdot \mathcal{E}_{\mathrm{gd}}(t)$.

Theorem 3.5. Assume that $a=-c$ and $d=-e$. If $f, g \in L^{1}(\mathbb{R})$, then the element $f \underset{\mathbb{Q}}{\Omega_{4}} g$ below considered defines a convolution followed by its norm inequality and factorization identity:

$$
\begin{aligned}
& \left(f \underset{\mathbb{Q}}{\left.\underset{\otimes}{\Omega_{4}} g\right)(x)}:=\frac{\sqrt{|b|}\left[\mathcal{E}_{\mathrm{ch}}(x)\right]^{-1}}{2 \pi} \int_{\mathbb{R}} \int_{\mathbb{R}} \mathcal{E}_{\mathrm{gd}}(x-u-v)\left[\mathcal{E}_{\mathrm{ch}}(u) f(u)\right]\left[\mathcal{E}_{\mathrm{ch}}(v) g(v)\right] d u d v,\right. \\
& \left\|f \underset{\mathbb{Q}}{\underset{\Omega_{4}}{\otimes}} g\right\|_{1} \leq\|f\|_{1}\|g\|_{1}, \quad \mathbb{Q}(f \underset{\mathbb{Q}}{\otimes} g)(x)=\Omega_{4}(x)(\mathbb{Q} f)(x)(\mathbb{Q} g)(x) .
\end{aligned}
$$

Proof. The norm inequality can be obtained by proceeding similarly as in the previous theorems, and so we ignore this step in here. A first direct computation yields

$$
\begin{aligned}
& \Omega_{4}(x)(\mathbb{Q} f)(x)(\mathbb{Q} g)(x) \\
& =e^{-i\left(a x^{2}+d x\right)} e^{-\frac{|b|}{2} x^{2}} \frac{1}{2 \pi} \int_{\mathbb{R}} \int_{\mathbb{R}} e^{i Q_{(a-e)}(x, u)} e^{i Q_{(a-e)}(x, v)} f(u) g(v) d u d v \\
& =e^{-i\left(a x^{2}+d x\right)} \sqrt{|b|} \frac{1}{\sqrt{2 \pi}} \int_{\mathbb{R}} e^{i b x t-\frac{|b|}{2} t^{2}} d t \frac{1}{2 \pi} \int_{\mathbb{R}} \int_{\mathbb{R}} e^{i Q_{(a-e)}(x, u)} e^{i Q_{(a-e)}(x, v)} f(u) g(v) d u d v \\
& =\frac{1}{2 \pi \sqrt{2 \pi}} \sqrt{|b|} \int_{\mathbb{R}} \int_{\mathbb{R}} \int_{\mathbb{R}} e^{i\left(a x^{2}+b x(u+v+t)+c u^{2}+c v^{2}+d x+e u+e v\right)} e^{-\frac{|b|}{2} t^{2}} f(u) g(v) d u d v d t .
\end{aligned}
$$

Then, considering $u=u, v=v$ and $s=u+v+t$, we obtain

$$
\begin{aligned}
& \Omega_{4}(x)(\mathbb{Q} f)(x)(\mathbb{Q} g)(x)=\frac{1}{2 \pi \sqrt{2 \pi}} \sqrt{|b|} \int_{\mathbb{R}} \int_{\mathbb{R}} \int_{\mathbb{R}} e^{i Q_{(a-e)}(x, s)} \times \\
& e^{i\left(c u^{2}+c v^{2}-c s^{2}+e u+e v-e s\right)-\frac{|b|}{2}(s-u-v)^{2}} f(u) g(v) d u d v d s \\
& =\frac{1}{\sqrt{2 \pi}} \int_{\mathbb{R}} e^{i Q_{(a-e)}(x, s)}\left\{\frac{\sqrt{|b|}\left[\mathcal{E}_{\mathrm{ch}}(s)\right]^{-1}}{2 \pi} \int_{\mathbb{R}} \int_{\mathbb{R}} \mathcal{E}_{\mathrm{gd}}(s-u-v) \times\right. \\
& \left.\left[\mathcal{E}_{\mathrm{ch}}(u) f(u)\right]\left[\mathcal{E}_{\mathrm{ch}}(v) f(v)\right] d u d v\right\} d s \\
& =\frac{1}{\sqrt{2 \pi}} \int_{\mathbb{R}} e^{i Q_{(a-e)}(x, s)}\left(f \underset{\mathbb{Q}}{\stackrel{\Omega_{4}}{\mathbb{Q}}} g\right)(s) d s=\mathbb{Q}(f \underset{\mathbb{Q}}{\stackrel{\Omega}{\otimes}} g)(x) .
\end{aligned}
$$

The proof of the theorem is complete.

Remark 3.6. Similarly to what was mentioned in Remark [3.2, we can see that (3.3)(3.5) take the form of other convolutions associated with the Fourier transform, fractional Fourier transform, and linear canonical transform, in corresponding special cases, when $\mathbb{Q}$ assumes the form of the Fourier transform, fractional Fourier transform, and linear canonical transform, respectively. 


\section{Applications}

In this final section, we exemplify some possibilities of application for the convolutions and integral operators considered above. In fact, we will obtain new Young type inequalities, norm decay rates of oscillatory integrals and solutions of convolution integral equations.

\subsection{Young Type Convolution Inequalities}

In this subsection, we will obtain certain norm inequalities for the convolutions (3.2)-(3.5) in a very general framework. For this purpose, we recall the Minkowski's integral inequality

$$
\left[\int_{\Theta_{2}}\left|\int_{\Theta_{1}} F(x, y) d \mu_{1}(x)\right|^{s} d \mu_{2}(y)\right]^{\frac{1}{s}} \leq \int_{\Theta_{1}}\left(\int_{\Theta_{2}}|F(x, y)|^{s} d \mu_{2}(y)\right)^{\frac{1}{s}} d \mu_{1}(x),
$$

where we have two measure spaces $\left(\Theta_{1}, \mu_{1}\right)$ and $\left(\Theta_{2}, \mu_{2}\right)$ and a measurable function $F(\cdot, \cdot)$ : $\Theta_{1} \times \Theta_{2} \longrightarrow \mathbb{C}$. Let $1 \leq p, q, r \leq \infty$ satisfy

$$
\frac{1}{p}+\frac{1}{q}=\frac{1}{r}+1
$$

The Banach spaces involved here are $L^{p}(\mathbb{R}), L^{q}(\mathbb{R}), L^{r}(\mathbb{R})$. For shortening the notation below, let us use the common symbol $\circledast$ for the four notations previously used: $\star, \otimes, \odot, \bigotimes$. We shall prove that:

$$
\begin{aligned}
& \|f \circledast g\|_{r} \leq C_{1}\|f\|_{p}\|g\|_{q}, \text { provided } f \in L^{p}(\mathbb{R}), g \in L^{q}(\mathbb{R}) ; \\
& \|f \circledast g\|_{s} \leq C_{2}\|f\|_{1}\|g\|_{1} \text { for any } s \geq 1, \text { provided } f, g \in L^{1}(\mathbb{R}),
\end{aligned}
$$

where $C_{1}, C_{2}$ are some positive constants. In here we would like to emphasize the power of the last inequality which is valid for any $s$, and so it is very different from the classic cases.

Let us prove those inequalities only for the convolution (3.5), and omit that ones for (3.2), (3.3), (3.4) and (3.5), since the proofs are analogous. A key point in all the proofs arises from the rapid decreasing behaviour of the Gaussian function $\mathcal{E}_{\text {gd }}$.

Proof of inequality (4.2). By changing the variable $t:=u+v$, we have

$$
\begin{aligned}
h(x): & =\left(f \underset{\mathbb{Q}}{\underset{\mathbb{Q}}{\Omega_{4}}} g\right)(x) \\
& =\frac{\sqrt{|b|}\left[\mathcal{E}_{\mathrm{ch}}(x)\right]^{-1}}{2 \pi} \int_{\mathbb{R}} \int_{\mathbb{R}} \mathcal{E}_{\mathrm{gd}}(x-u-v)\left[\mathcal{E}_{\mathrm{ch}}(u) f(u)\right]\left[\mathcal{E}_{\mathrm{ch}}(v) f(v)\right](v) d u d v \\
& =\frac{\sqrt{|b|}\left[\mathcal{E}_{\mathrm{ch}}(x)\right]^{-1}}{2 \pi} \int_{\mathbb{R}} \mathcal{E}_{\mathrm{gd}}(x-t) d t\left(\int_{\mathbb{R}}\left[\mathcal{E}_{\mathrm{ch}}(t-v) f(t-v)\right]\left[\mathcal{E}_{\mathrm{ch}}(v) g(v)\right] d v\right)
\end{aligned}
$$




$$
=\frac{\sqrt{|b|}\left[\mathcal{E}_{\mathrm{ch}}(x)\right]^{-1}}{2 \pi} \int_{\mathbb{R}} \mathcal{E}_{\mathrm{gd}}(s-t) F(t) d t
$$

where

$$
F(t):=\int_{\mathbb{R}}\left[\mathcal{E}_{\mathrm{ch}}(t-v) f(t-v)\right]\left[\mathcal{E}_{\mathrm{ch}}(v) g(v)\right] d v .
$$

Evidently, $\mathcal{E}_{\text {ch }} f \in L^{p}(\mathbb{R}), \mathcal{E}_{\text {ch }} g \in L^{q}(\mathbb{R})$. Applying the well-known Young's convolution inequality for the classic case [4, 5] gives $F \in L^{r}(\mathbb{R})$. Remind that $\left|\mathcal{E}_{\mathrm{ch}}^{-1}(x)\right|=1$, and $\mathcal{E}_{\mathrm{gd}} \in$ $L^{1}(\mathbb{R})$. Again, applying Young's inequality for the case $\frac{1}{r}+\frac{1}{1}=\frac{1}{r}+1$, we derive that the function defined by the right-hand side of (4.4) belongs to $L^{r}(\mathbb{R})$. This means that $h \in L^{r}(\mathbb{R})$.

Proof of inequality (4.3). Since $\mathcal{E}_{\text {gd }}$ is a rapidly decreasing function, $\mathcal{E}_{\text {gd }} \in L^{s}(\mathbb{R})$ for any $s \geq 1$, and

$$
\int_{\mathbb{R}}\left|\mathcal{E}_{\text {gd }}( \pm x \pm u \pm v)\right|^{s} d x=\left\|\mathcal{E}_{\text {gd }}\right\|_{s}^{s} \quad(u, v \text { are fixed in } \mathbb{R}) .
$$

Applying (4.1) gives

$$
\begin{aligned}
& {\left[\int_{\mathbb{R}}\left|\int_{\mathbb{R}^{2}} \mathcal{E}_{\mathrm{gd}}(x-v-u) f(u) g(v) d u d v\right|^{s} d x\right]^{1 / s}} \\
& \quad \leq \int_{\mathbb{R}^{2}}\left(\int_{\mathbb{R}}\left|\mathcal{E}_{\mathrm{gd}}(x-v-u)\right|^{s}|f(u)|^{s}|g(v)|^{s} d x\right)^{1 / s} d u d v \\
& \quad=\int_{\mathbb{R}^{2}}\left(\int_{\mathbb{R}}\left|\mathcal{E}_{\mathrm{gd}}(x-v-u)\right|^{s} d x\right)^{1 / s}|f(u)||g(v)| d u d v \\
& \quad=\left\|\mathcal{E}_{\mathrm{gd}}\right\|_{s} \int_{\mathbb{R}^{2}}|f(u)||g(v)| d u d v=\left\|\mathcal{E}_{\mathrm{gd}}\right\|_{s} 2 \pi\|f\|_{1}\|g\|_{1} .
\end{aligned}
$$

We thus obtain inequality (4.3).

There is a notable difference on the image and domain spaces between the here proposed convolutions and previously constructed convolutions, associated with the fractional Fourier transform and the linear canonical transform, as indicated by the theorem below.

Theorem 4.1. The convolutions (3.2), (3.3), (3.4), and (3.5), possess their Young's convolution inequalities given by (4.2) and (4.3).

In other words, if $f \in L^{p}(\mathbb{R})$ and $g \in L^{q}(\mathbb{R})$, then each one of the proposed convolutions defines a new function in $L^{r}(\mathbb{R})$, where $1 / p+1 / q=1 / r+1$. Moreover, if $f, g \in L^{1}(\mathbb{R})$, then each one of those convolutions also defines a function in $L^{s}(\mathbb{R})$, for any $s \geq 1$.

Remark 4.2. (a) Using a direct notation, we may write:

$$
L^{p}(\mathbb{R}) \circledast L^{q}(\mathbb{R}) \subseteq L^{r}(\mathbb{R}), \text { where } \frac{1}{p}+\frac{1}{q}=\frac{1}{r}+1 ;
$$




$$
L^{1}(\mathbb{R}) \circledast L^{1}(\mathbb{R}) \subseteq L^{s}(\mathbb{R}), \text { for any } s \geq 1 .
$$

Letting $p=q=r=1$ in inclusion (4.5), or let $s=1$ in inclusion (4.6), we retrieve the norm inequalities proved by Theorems 3.1, 3.3, 3.4, and 3.5, with the explicit constant $C_{1}=C_{2}=1$ (cf. inequalities (4.2)-(4.3)).

(b) Choosing $s=2$ in (4.3), we see that if $f, g \in L^{1}(\mathbb{R})$, then the convolution defines a function in the space $L^{1}(\mathbb{R}) \cap L^{2}(\mathbb{R})$. This result is in accordance with the known circumstance that a convolution $f * g$ inherits the best properties of both $f$ and $g$. In particular, since a convolution can be seen as a filtering, averaging, inner product and as, somehow, a smoothing action, a Young type convolution inequality (4.3) is a striking feature for new convolutions. Having in mind that in the literature only the corresponding situation for the Fourier case is known, inequality (4.3) exhibits a remarkable property associated with the proposed convolutions.

(c) Even in the case of the classical Fourier convolution, one has only the Young's convolution inequality (4.2), and (4.3) for $s=1$. Therefore, the Young's inequality (4.3) is a specific characteristic of the convolutions here introduced.

The following corollary is an immediate consequence of the above theorem.

Corollary 4.3. The Banach space $L^{1}(\mathbb{R})$, equipped with each one of convolutions (3.2), (3.3), (3.4), and (3.5), becomes a normed ring.

\subsection{Norm Decay Rate of Oscillatory Integrals}

The general oscillatory integral theory has its origins at the heart of Harmonic Analysis, in which the Fourier's case is the original and probably the best example of an oscillatory integral, and leads us to consider more general oscillatory integrals. In recent years, there have been many efforts for estimating norm decay rates of Fourier oscillatory integrals (see e.g. [19, 20, 23, 27] and references therein).

The possible best norm decay rate of the quadratic-phase Fourier integral operator $\mathbb{Q}$ can be seen as an immediate consequence of the identity (2.2). So, in a more global way, let us consider the quadratic-phase Fourier oscillatory integral operator

$$
\left(\mathbb{T}_{\lambda} \phi\right)(x):=\int_{\mathbb{R}} e^{i \lambda Q_{(a-e)}(x, y)} \psi(x, y) \phi(y) d y,
$$

where $Q_{(a-e)}(x, y)$ defined by (1.1) is called the phase, and $\psi(x, y)$ being a smooth compactly supported function on $\mathbb{R}^{2}$ is said to be amplitude (cf. [19, 20, 23, 27]). Recall that $b \neq 0$. 
The idea is to understand the behaviour of the norm of $\mathbb{T}_{\lambda}$ when $\lambda$ is varying through $\mathbb{R}$. The case of $\lambda=0$ is obvious (as a degenerated case) and so it is omitted (in fact, it is even enough to consider $\lambda>0$ ).

Theorem 4.4. $\mathbb{T}_{\lambda}$ can be extended to a bounded linear operator defined in $L^{2}(\mathbb{R})$ with norm

$$
\left\|\mathbb{T}_{\lambda}\right\|_{2} \leq \frac{C}{\sqrt{|\lambda|}}
$$

where $C$ is independent on $\lambda$.

Proof. Let $M \subset \mathbb{R}^{2}$ denote the compact support of $\psi$, and let $\chi_{M}(x)$ and $\chi_{M}(y)$ stand for the characteristic functions with variable $x$ and $y$, respectively. It is easily seen that $\mathbb{T}_{\lambda} \phi \in L^{2}(\mathbb{R})$, provided $\phi \in L^{2}(\mathbb{R})$. Indeed, having in mind that $\psi(x, y)$ is uniformly bounded on $M \times M$ (there is a constant $C$ such that $|\psi(x, y)| \leq C<\infty$ ), and using (4.1) and the Cauchy-Bunyakovsky-Schwarz inequality, we have

$$
\begin{aligned}
\int_{\mathbb{R}}\left|\left(\mathbb{T}_{\lambda} \phi(x)\right)\right|^{2} d x & =\int_{\mathbb{R}} d x\left|\int_{\mathbb{R}} e^{i \lambda Q_{(a-e)}(x, y)} \chi_{M}(x) \chi_{M}(y) \psi(x, y) \phi(y) d y\right|^{2} \\
\leq & {\left[\int_{\mathbb{R}} \chi_{M}(y)|\phi(y)| d y(\underbrace{\int_{\mathbb{b}_{M}} \chi_{M}(x)|\psi(x, y)|^{2} d x}_{\mathbb{R}})^{1 / 2}\right]^{2} } \\
& \leq C\left(\int_{\mathbb{R}} \chi_{M}(y)|\phi(y)| d y\right)^{2} \leq C\left(\int_{M} \chi_{M}(y) d y\right) \cdot\left(\int_{M}|\phi(y)|^{2} d y\right)<\infty
\end{aligned}
$$

We shall prove the decay rate. By the assumption, $\psi(x, y)$ can be seen as an $L^{2}$-integrable function with variable $y \in \mathbb{R}$ for $x \in M$ fixed, by $\psi(x, y)=\chi_{M}(y) \cdot \psi(x, y)$ for $y \in \mathbb{R}$. Moreover, for any $f \in L^{2}(\mathbb{R}), \psi(x, y) \cdot f(y)$ is also $L^{2}(\mathbb{R})$-integrable in the variable $y \in \mathbb{R}$, for $x \in M$ fixed, as

$$
\int_{\mathbb{R}}\left|\chi_{M}(y) \psi(x, y) f(y)\right|^{2} d y=\int_{\mathbb{R}}\left|\chi_{M}(y) \psi(x, y)\right|^{2}|f(y)|^{2} d y \leq C \int_{\mathbb{R}}|f(y)|^{2} d y<\infty .
$$

Therefore, we can write $\left(\mathbb{T}_{\lambda} f\right)(x)=\left(\mathbb{Q}_{\lambda(a-e)} \psi f\right)(x)$ where $\mathbb{Q}_{\lambda(a-e)}$ is defined in the same way as $\mathbb{Q}_{(a-e)}$ by (1.2), but with the phase $\lambda Q_{(a-e)}(x, y)$. We can use (2.2) and apply the Minkowski and Cauchy-Bunyakovsky-Schwarz inequalities to have

$$
\begin{aligned}
\left\|\left(\mathbb{T}_{\lambda} f\right)(x)\right\|_{2}^{2} & =\left\|\left(\mathbb{Q}_{\lambda(a-e)} \psi f\right)(x)\right\|_{2}^{2}=\frac{1}{|b \lambda|}\|\langle(\psi f)(y),(\psi f)(y)\rangle\|_{2}^{2} \\
& =\frac{1}{|b \lambda|} \int_{\mathbb{R}} d x\left|\int_{\mathbb{R}} \chi_{M}(x) \chi_{M}(y) \psi(x, y) f(y) \overline{\psi(x, y) f(y)} d y\right|^{2} \\
& \leq \frac{1}{|b \lambda|} \int_{\mathbb{R}} \chi_{M}(y)|f(y)|^{2} d y\left(\int_{\mathbb{R}} \chi_{M}(x)|\psi(x, y)|^{4} d x\right)^{1 / 2} \leq \frac{C}{|\lambda|}\|f\|_{2}^{2} .
\end{aligned}
$$

Therefore, we have the desired decay rate. 


\subsection{Solvability of Integral Equations}

In view to exemplify other applicability of our previously presented convolutions, we will now consider some classes of integral equations associated with the convolutions proposed in Theorems 3.1-3.5. This will illustrate one of the possible applications of the above-proved theorems. We recall that we are using $\circledast$ for denoting any of the previously introduced convolutions: $\star, \otimes, \odot, \bigotimes$. Consider the convolution equation

$$
\lambda \varphi(x)+(k \circledast \varphi)(x)=p(x),
$$

where $\lambda \in \mathbb{C}, k, p$ are given in $L^{1}(\mathbb{R})$, and $\varphi$ is to be determined there. In equation (4.8), when the convolution $\circledast$ is taken one of the possibilities (3.2), (3.3), (3.4), or (3.5), then let us also use $\Omega^{*}$ to be the corresponding function in

$$
\left\{\Omega_{1}, \Omega_{2}, \Omega_{3}, \Omega_{4}\right\}
$$

respectively. We now shall use the notation $S(x):=\lambda+\Omega^{*}(x) \cdot(\mathbb{Q} k)(x)$.

Theorem 4.5. Assume that $S(x) \neq 0$ for every $x \in \mathbb{R}$ and $\frac{\mathbb{Q k}}{S} \in L^{1}(\mathbb{R})$. Equation (4.8) has a solution in $L^{1}(\mathbb{R})$ if and only if

$$
\mathbb{Q}^{-1}\left(\frac{\mathbb{Q} k}{S}\right) \in L^{1}(\mathbb{R})
$$

Moreover, if the condition (4.9) holds, then the solution of (4.8) is given in explicit form by $\varphi=\mathbb{Q}^{-1}\left(\frac{\mathbb{Q} k}{S}\right) \in L^{1}(\mathbb{R})$.

Proof. Necessity. Suppose that equation (4.8) has a solution $\varphi \in L^{1}(\mathbb{R})$. Applying $\mathbb{Q}$ to both sides of (4.8), we obtain

$$
\lambda(\mathbb{Q} \varphi)(x)+\Omega^{*}(x)(\mathbb{Q} \varphi)(x)(\mathbb{Q} k)(x)=(\mathbb{Q} p)(x),
$$

i.e., $S(x)(\mathbb{Q} \varphi)(x)=(\mathbb{Q} p)(x)$. Having in mind that $S(x) \neq 0$ for all $x \in \mathbb{R}$, we get $\mathbb{Q} \varphi=\frac{\mathbb{Q} p}{S}$. Taking profit that $\frac{\mathbb{Q} p}{S} \in L^{1}(\mathbb{R})$, we receive

$$
\varphi=\mathbb{Q}^{-1}\left(\frac{\mathbb{Q} p}{S}\right) \in L^{1}(\mathbb{R}) .
$$

Sufficiency. Let $\varphi=\mathbb{Q}^{-1}\left(\frac{\mathbb{Q} p}{S}\right) \in L^{1}(\mathbb{R})$. By $\varphi \in L^{1}(\mathbb{R})$ we get $S(x)(\mathbb{Q} \varphi)(x)=(\mathbb{Q} p)(x)$. Using the factorization identity of the convolution, we obtain

$$
\mathbb{Q}(\lambda \varphi(x)+k \circledast \varphi)=(\mathbb{Q} p)(x) .
$$

Thanks to the uniqueness theorem of $\mathbb{Q}$, we conclude that $\varphi(x)$ fulfills equation (4.8) for almost every $x \in \mathbb{R}$. The theorem is proved. 


\section{Acknowledgments}

The first-named author was supported in part by FCT-Portuguese Foundation for Science and Technology through the Center for Research and Development in Mathematics and Applications (CIDMA) at Universidade de Aveiro, within the project UID/MAT/04106/2013. The two last named-authors were partially supported by the Viet Nam National Foundation for Science and Technology Development (NAFOSTED).

\section{References}

[1] Almeida, L.B.: Product and convolution theorems for the fractional Fourier transform. IEEE Signal Process. Lett. 4(1), 15-17 (1997)

[2] Anh, P.K., Castro, L.P., Thao, P.T., Tuan, N.M.: Two new convolutions for the fractional Fourier transform. Wireless Pers. Commun. 92(2), 623-637 (2017)

[3] Anh, P.K., Tuan, N.M., Tuan, P.D.: The finite Hartley new convolutions and solvability of the integral equations with Toeplitz plus Hankel kernels. J. Math. Anal. Appl. 397(2), $537-549(2013)$

[4] Beckner, W.: Inequalities in Fourier analysis. Ann. Math. 102, 159-182 (1975)

[5] Brascamp, H.J., Lieb, E.H.: Best constants in Young's inequality, its converse, and its generalization to more than three functions. Adv. Math. 20, 151-173 (1976)

[6] Castro, L.P.: Regularity of convolution type operators with PC symbols in Bessel potential spaces over two finite intervals, Math. Nachr. 261-262, 23-36 (2003)

[7] Castro, L.P., Duduchava, R., Speck, F.-O.: Finite interval convolution operators with transmission property, Integral Equations Oper. Theory 52(2), 165-179 (2005)

[8] Castro, L.P., Haque, M.R., Murshed, M.M., Saitoh, S., Tuan, N.M.: Quadratic Fourier transforms. Ann. Funct. Anal. AFA 5(1), 10-23 (2014)

[9] Castro, L.P., Kapanadze, D.: Dirichlet-Neumann-impedance boundary value problems arising in rectangular wedge diffraction problems. Proc. Am. Math. Soc. 136(6), 2113$2123(2008)$ 
[10] Castro, L.P., Kapanadze, D.: Wave diffraction by a half-plane with an obstacle perpendicular to the boundary. J. Differ. Equations 254(2), 493-510 (2013)

[11] Castro, L.P., Speck, F.-O.: Inversion of matrix convolution type operators with symmetry. Port. Math. (N.S.) 62(2), 193-216 (2005)

[12] Castro, L.P., Saitoh, S.: New convolutions and norm inequalities. Math. Inequal. Appl. 15(3), 707-716 (2012)

[13] Castro, L.P., Saitoh, S., Tuan, N.M.: Convolutions, integral transforms and integral equations by means of the theory of reproducing kernels. Opusc. Math. 32(4), 633-646 (2012)

[14] Jain, P., Jain, S.: On Young type inequalities for generalized convolutions. Proc. A. Razmadze Math. Inst. 164, 45-61 (2014)

[15] Jain, P., Jain, S.: Generalized convolution inequalities and application. Mediterr. J. Math. 14(4) (2017). https://doi.org/10.1007/s00009-017-0961-3

[16] Jain, P., Jain, S., Kumar, R.: On fractional convolutions and distributions. Integral Transforms Spec. Funct. 26(10), 885-899 (2015)

[17] Nhan, N.D.V., Duc, D.T.: Weighted $L^{p}$-norm inequalities in convolutions and their applications. J. Math. Inequal. 2, 45-55 (2008)

[18] Oinarov, R.: Boundedness and compactness of a class of convolution integral operators of fractional integration type. Proc. Steklov Inst. Math. 293, 255-271 (2016); translation from Tr. Mat. Inst. Steklova 293, 263-279 (2016)

[19] Phong, D.H., Stein, E.M.: Models of degenerate Fourier integral operators and Radon transforms. Ann. Math. 140, 703-722 (1994)

[20] Phong, D.H., Stein, E.M.: Oscillatory integrals with polynomial phases. Invent. Math. 110, 39-62 (1992)

[21] Rudin, W.: Functional Analysis (second ed.). McGraw-Hill, New York (1991)

[22] Singh, A.K., Saxena, R.: On convolution and product theorems for FRFT. Wireless Pers. Commun. 65(1), 189-201 (2012)

[23] Stein, E.M.: Harmonic Analysis. Princeton Univ. Press, Princeton, New Jersey (1993) 
[24] Titchmarsh, E.C.: Introduction to the Theory of Fourier Integrals (third ed.). Chelsea Publishing Co., New York (1986)

[25] Tuan, N.M., Huyen, N.T.T.: Applications of generalized convolutions associated with the Fourier and Hartley transforms. J. Integral Equations Appl. 24(1), 111-130 (2012)

[26] Vinogradov, O.L.: Sharp inequalities for approximations of convolution classes on the real line as the limit case of inequalities for periodic convolutions. Sib. Math. J. 58(2), 190-204 (2017); translation from Sib. Mat. Zh. 58(2), 251-269 (2017)

[27] Wright, J.: From oscillatory integrals to complete exponential sums. Math. Res. Lett. 18(2), 231-250 (2011)

[28] Zayed, A.I.: A convolution and product theorem for the fractional Fourier transform. IEEE Signal Process. Lett. 5(4), 102-103 (1998)

L.P. Castro

CIDMA - Center for Research and Development in Mathematics and Applications, Department of Mathematics, University of Aveiro, 3810-193 Aveiro, Portugal

e-mail: castro@ua.pt

L.T. Minh

Department of Mathematics, Hanoi Architectural University, Hanoi, Vietnam

e-mail: tienminh.hau@gmail.com

N.M. Tuan

Department of Mathematics, College of Education, Vietnam National University, Hanoi, Vietnam e-mail: tuannm@hus.edu.vn 